\title{
THE GALACTIC CENTRE AT 408 MHz
}

\author{
A. G. LITTLE \\ University of Sydney, Sydney, Australia
}

\begin{abstract}
The Molonglo radio telescope has been used to map the galactic centre region with a resolution of 2.9'. Although similar to microwave maps of comparable resolution, there are differences. In particular, the source G1.05-0.1 is not visible at microwaves, and the source G0.1-0.0 is not visible at $408 \mathrm{MHz}$. The flux values of all the other sources around Sgr A confirm that they are thermal and that the sources G0.7-0.0, G0.5 -0.0, and G0.2-0.0 are optically thick.

A more extensive galactic background survey with the cross shows the existence of a peak in the background emission with a brightness temperature of about $500 \mathrm{~K}$ at the galactic centre. This is probably the nonthermal source observed at $85 \mathrm{MHz}$. The higher-resolution observations of this map show the narrower, hotter region which appears on the microwave map and which surrounds Sgr A. This has a brightness temperature of $2100 \mathrm{~K}$, which cannot be accounted for by a simple thermal spectrum extrapolated from microwaves.
\end{abstract}

Although there are now many occultation measurements of Sgr A at low frequencies, very little data is available for the remainder of the region below about $1.4 \mathrm{GHz}$. Moreover, what high-resolution information is available is confined to frequencies above a few GHz. The Molonglo radio telescope (Mills et al., 1963) operating at $408 \mathrm{MHz}$ should therefore be able to add useful knowledge of temperatures and flux densities of this very complex region. If we confine the comparisons with the microwave results to the high-resolution maps of Broten et al. (1965), Downes et al. (1966), and Hollinger (1965), which have been made with beamwidths similar to that of the Molonglo telescope $\left(2.9^{\prime}\right)$, direct comparisons can be made without the uncertainties introduced by different resolving powers.

The line scans corresponding to these observations are shown in Figure 1. The side lobes associated with the strong source Sgr A can be seen, and some correction for these has been attempted by making use of the line scans for interpolation.

The resulting contour map is shown in Figure 2. The unit contour interval is $680 \mathrm{~K}$. which is in terms of the main beam brightness temperature. This has been derived from the flux calibration using the Rayleigh-Jeans relation between flux and temperature. The lowest contour corresponds to a temperature of $1075 \mathrm{~K}$, which was determined from a survey of the galactic plane made by Green (1974) using the Molonglo cross. Two sources were used for both flux and position calibration: PKS 1643-22 and PKS 1814-51. The data for these sources are shown in Table I. The fluxes were based on the Wyllie (1969a, 1969b) scale, and the above positions and fluxes are from Hunstead (1972).

A comparison with the microwave maps shows that with the exception of the source G0.1 - 0.0 all the sources listed at microwaves appear on the $408 \mathrm{MHz}$ map. An additional source is seen at $408 \mathrm{MHz}$ which appears only as a suggestion in the contours of the source G1.1 -0.1 at microwaves. This new source, which has a flux comparable to G1.1 -0.1 , is thus clearly nonthermal in character and has been designated G1.05-0.1 after Downes and Maxwell (1966). 

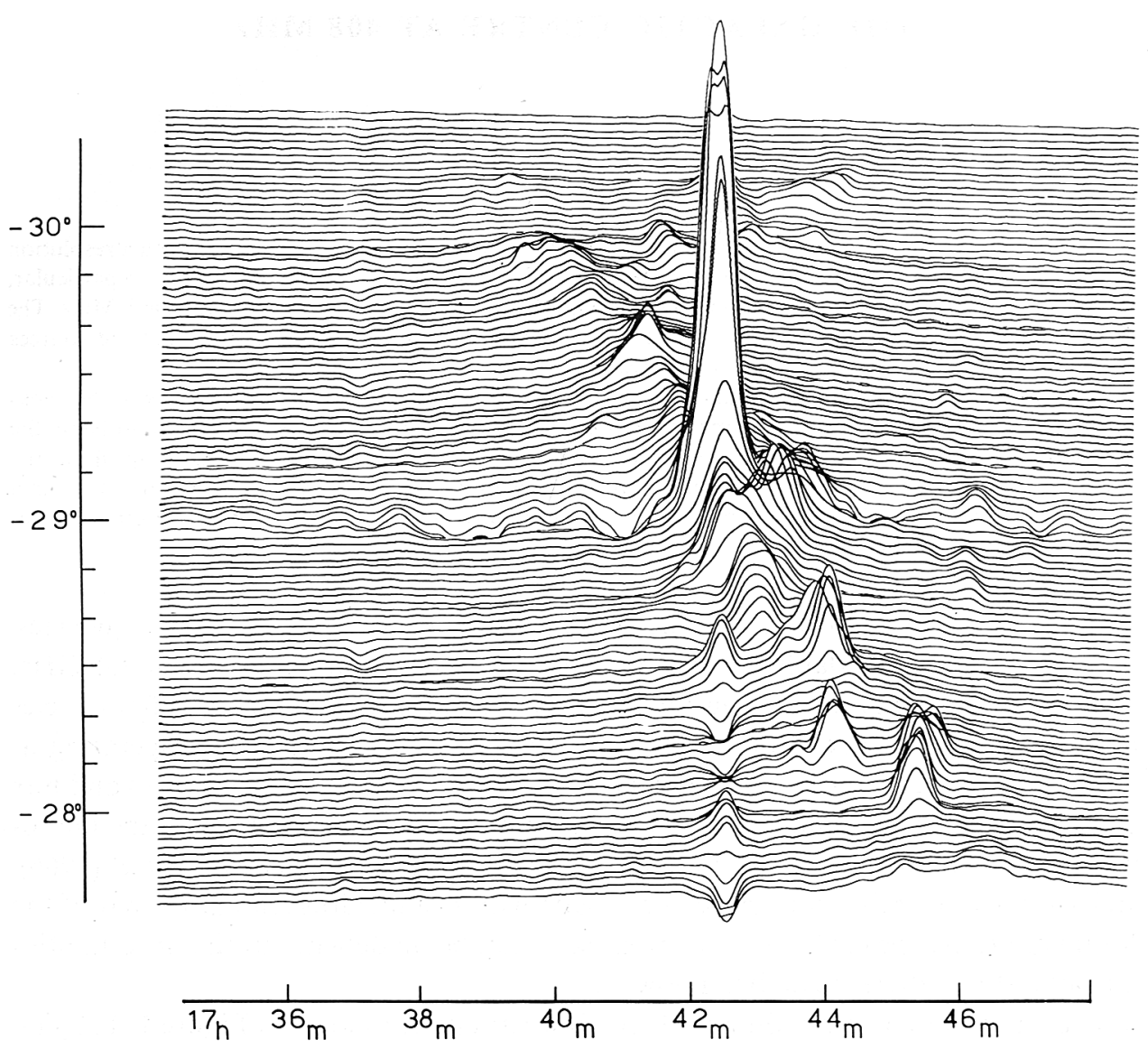

Fig. 1. (1) Line scans of the galactic centre at $408 \mathrm{MHz}$ (Sgr A is saturated at this sensitivity).

The integrated flux densities for all the sources are given in Table II together with values for the three microwave maps taken from Downes and Maxwell (1966).

It can be seen that the present observations support the view that apart from $\mathrm{Sgr} \mathrm{A}$ and G1.0 - 0.1 all the sources are thermal in character. Moreover, the sources G0.2-0.0, G0.5 - 0.0 and G0.7 -0.0 appear to be optically thick at $408 \mathrm{MHz}$. The fact that G0.1-0.0 is not visible may be due to the source having a brightness temperature close to that of the hot background on which it is located.

In determining the flux densities there was the usual problem of separating the sources from the background. This has been done here by using the line scans, but it is by no means a precise operation. The temperatures of the background levels so determined are shown in the last column of Table II. The resulting uncertainty in the flux density values is of the order of $25 \%$.

A value for the peak brightness temperature for each source has also been obtained and is given in Table III. However, because of the smoothing introduced by the beam, 


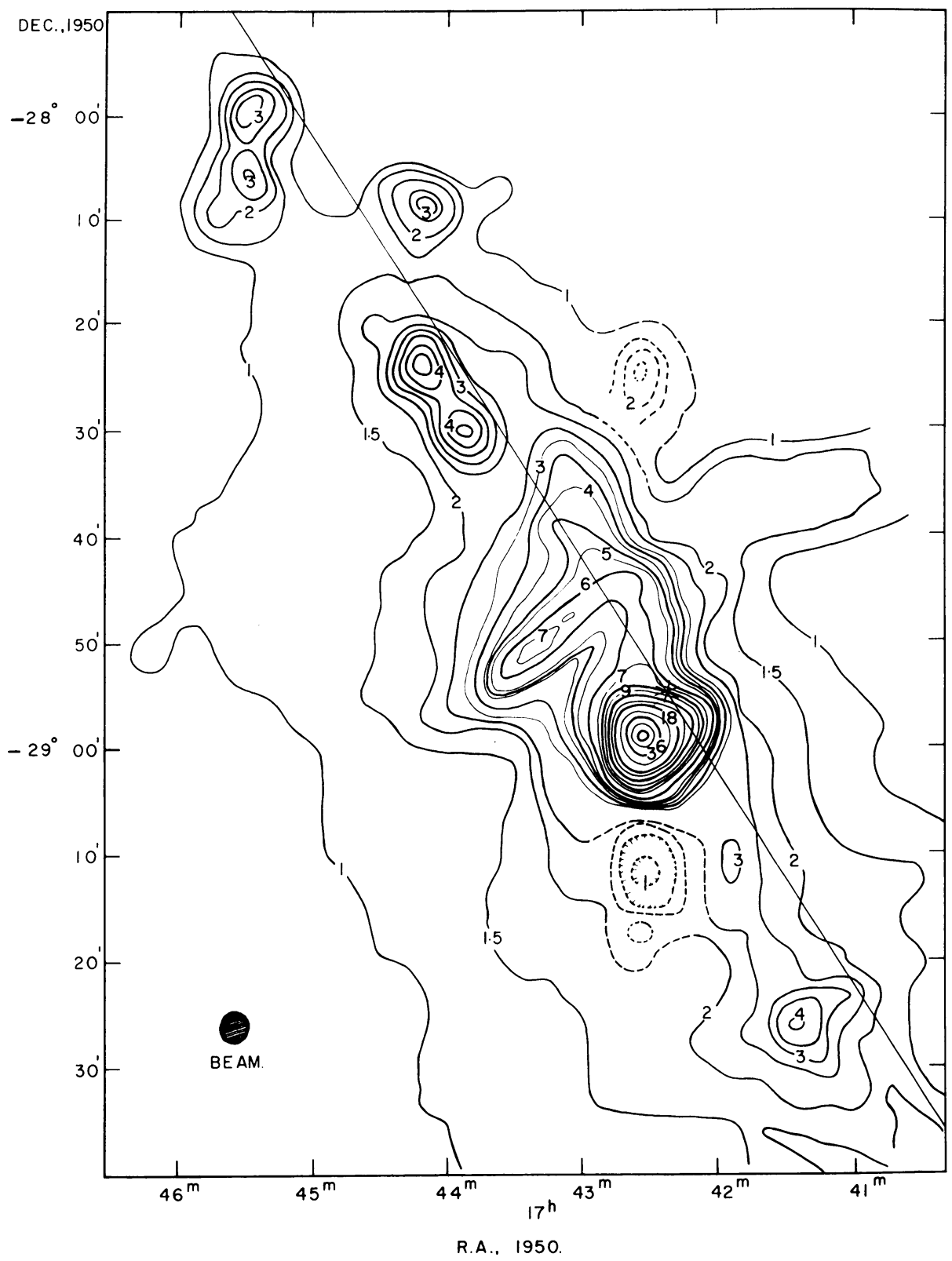

Fig. 2. Contour map of the galactic centre region at $408 \mathrm{MHz}$. The lowest contour is $1075 \mathrm{~K}$ and the unit contour interval is $680 \mathrm{~K}$. 
TABLE I

Flux and position calibration sources

\begin{tabular}{lcll}
\hline Source & Flux (Jy) & R.A. (1950) & Dec. (1950) \\
\hline PKS 1643-22 & $5.46 \pm 0.09$ & $16^{\mathrm{h}} 43^{\mathrm{m}} 04^{\mathrm{s}} 8 \pm 0.09$ & $-22^{\circ} 22^{\prime} 35^{\prime \prime} .1 \pm 1^{\prime \prime} 4$ \\
PKS $1814-51$ & $13.6 \pm 0.2$ & $18^{\mathrm{h}} 14^{\mathrm{m}} 07^{\mathrm{s}} 81 \pm 0.14$ & $-51^{\circ} 59^{\prime} 22^{\prime \prime} .1 \pm 1^{\prime \prime} 6$ \\
\hline
\end{tabular}

TABLE II

Source flux densities

\begin{tabular}{|c|c|c|c|c|c|}
\hline \multirow[t]{2}{*}{ Source } & \multicolumn{4}{|l|}{ Flux (Jy) } & \multirow{2}{*}{$\begin{array}{l}\text { Background } \\
\text { temp. at } \\
0.408 \mathrm{GHz}(\mathrm{K})\end{array}$} \\
\hline & $14.5 \mathrm{GHz}$ & $8.0 \mathrm{GHz}$ & $5.0 \mathrm{GHz}$ & $0.408 \mathrm{GHz}$ & \\
\hline G359.4-0.1 & 11 & 14 & 16 & 9.8 & 2095 \\
\hline G0.1-0.0 & 60 & 60 & 70 & - & 3115 \\
\hline $\mathrm{G} 0.2-0.0$ & 140 & 120 & 150 & 40 & 3115 \\
\hline G0.5-0.0 & 37 & 41 & 52 & 9.2 & 2095 \\
\hline G0.7-0.0 & 45 & 58 & 58 & 9.7 & 2095 \\
\hline $\mathrm{G} 0.9+0.1$ & - & 14 & 15 & 10 & 1211 \\
\hline G1.05-0.1 & - & - & - & 15.5 & 1075 \\
\hline $\mathrm{G} 1.1-0.1$ & 20 & 20 & 20 & 15.2 & 1075 \\
\hline
\end{tabular}

these are low, and a simple correction using the chord construction of Bracewell (1955) has been applied. This corrected value is also given in Table III with the estimated error in the final temperatures.

For the optically thick sources it should be possible to make some estimate of the electron temperature. However, in order to do this, corrections have to be made for the background and foreground emission which are not negligible in this region. For example, the source $\mathrm{G} 0.2-0.0$ has a peak brightness temperature of $6400 \mathrm{~K}$ but is located on a background of $3115 \mathrm{~K}$. Depending on whether the source is located in front of or behind the background and the relative amount of thermal and nonthermal radiation in the background, the correction to be applied to the observed peak temperatures to determine a value for the electron temperature could be quite substantial. It is beyond the scope of the present paper to discuss this.

The positions and angular sizes for the sources are given in Table IV, where the microwave values from Downes and Maxwell (1966) are indicated in parentheses. There are slight differences in position between the two, which may be simply related to the greater optical depth at $408 \mathrm{MHz}$ since the sources are not symmetrical.

The measured value of flux density, position and angular size for Sgr A together with occultation values (Maxwell and Taylor, 1968) at $405 \mathrm{MHz}$ are given in Table V. The present observations agree very well with the occultation results. For this source the background contour level was determined to be 7 units ( $5255 \mathrm{~K})$.

Finally, the background survey (Green, 1974) mentioned earlier shows an extended source at the galactic centre which has a peak temperature of about $500 \mathrm{~K}$ and which 
is roughly $2^{\circ}-3^{\circ}$ in angular dimensions. This is illustrated in Figure 3, which shows the temperature variation (a) along the galactic plane at latitude zero and (b) transverse to the plane at longitude zero. The temperatures are average values over $0.5^{\circ} \times 0.5^{\circ}$ with all sources removed. This peak is almost certainly due to the nonthermal source observed at $85 \mathrm{MHz}$ (Mills, 1956). At this frequency this source had two peaks of measured brightness temperature $34000 \mathrm{~K}$ with an absorption dip at

TABLE III

Peak brightness temperatures for $408 \mathrm{MHz}$ Sources

\begin{tabular}{rlc}
\hline Source & $\begin{array}{l}\text { Peak observed } \\
\text { brightness } \\
\text { temperature }(\mathrm{K})\end{array}$ & $\begin{array}{l}\text { Corrected peak } \\
\text { brightness } \\
\text { temperature }(\mathrm{K})\end{array}$ \\
\hline G359.4-0.1 & 3200 & $3500 \pm 300$ \\
G0.1-0.0 & 5600 & - \\
G0.2-0.0 & 5600 & $6400 \pm 600$ \\
G0.5-0.0 & 3500 & $3900 \pm 350$ \\
G0.7-0.0 & 3500 & $3800 \pm 350$ \\
G0.9+0.1 & 2600 & $3200 \pm 280$ \\
G1.05-0.1 & 2400 & $2700 \pm 230$ \\
G1.1-0.1 & 2500 & $2800 \pm 250$ \\
\hline
\end{tabular}

TABLE IV

Positions and angular sizes of $408 \mathrm{MHz}$ sources

\begin{tabular}{rlrlrl}
\hline Source & \multicolumn{2}{l}{$\begin{array}{l}\text { Right ascension } \\
(1950)\end{array}$} & $\begin{array}{l}\text { Declination } \\
(1950)\end{array}$ & $\begin{array}{l}\text { Angular dimensions } \\
\text { (arcmin) }\end{array}$ \\
\hline G359.4-0.1 & $17^{\mathrm{h}} 41^{\mathrm{m}}$ & $25 \mathrm{~s} 8(22)$ & $-29^{\circ} 26^{\prime} 0(26.6)$ & $5.3 \times 4.3(5 \times 3)$ \\
G0.1-0.0 & $17-$ & $(42)-(34)$ & $-(-28)-(51)$ & - & $(7 \times 5)$ \\
G0.2-0.0 & $1743(42) 19.7(59)$ & $-2850.4(47)$ & $16.3 \times 1.6(17 \times 5)$ \\
G0.5-0.0 & 1743 & $52.3(49)$ & $-2830.2(29.1)$ & $4.8 \times 3.2(8 \times 4)$ \\
G0.7-0.0 & 1744 & $11.2(7)$ & $-2823.9(21.6)$ & $4.9 \times 2.9(5 \times 2)$ \\
G0.9-0.1 & 1744 & $9.0(7)$ & $-288.7(7.6)$ & $3.8 \times 3.4(5 \times 5)$ \\
G1.05-0.1 & 1745 & 27.8 & -285.8 & $6.8 \times 3.9$ & - \\
G1.1-0.1 & 1745 & $28.8(25)$ & $-2759.5(58.2)$ & $5.7 \times 4$ & $(6 \times 6)$ \\
\hline
\end{tabular}

TABLE V

Data on Sgr A

\begin{tabular}{lcccc}
\hline $\begin{array}{l}\text { Frequency } \\
(\mathrm{MHz})\end{array}$ & $\begin{array}{l}\text { Flux } \\
(\mathrm{Jy})\end{array}$ & R.A. (1950) & Dec.(1950) & $\begin{array}{l}\text { Angular } \\
\text { dimensions } \\
\text { (arcmin) }\end{array}$ \\
\hline 408 & 229 & $17^{\mathrm{h}} 42^{\mathrm{m}} 32^{\mathrm{s}} .^{\mathrm{s}} 6 \pm 0.3$ & $-28^{\circ} 59^{\prime} \pm 3^{\prime \prime}$ & $2.6 \times 3.4$ \\
& & & \\
405 & Occultation measurements (Maxwell and Taylor, 1968) & \\
405 & 235 & 30.9 & $59^{\prime} 13^{\prime \prime}$ & $3.5 \times 4.4$ \\
& 225 & 32.2 & $58^{\prime} 51^{\prime \prime}$ & $2.6 \times 3.4$ \\
\hline
\end{tabular}




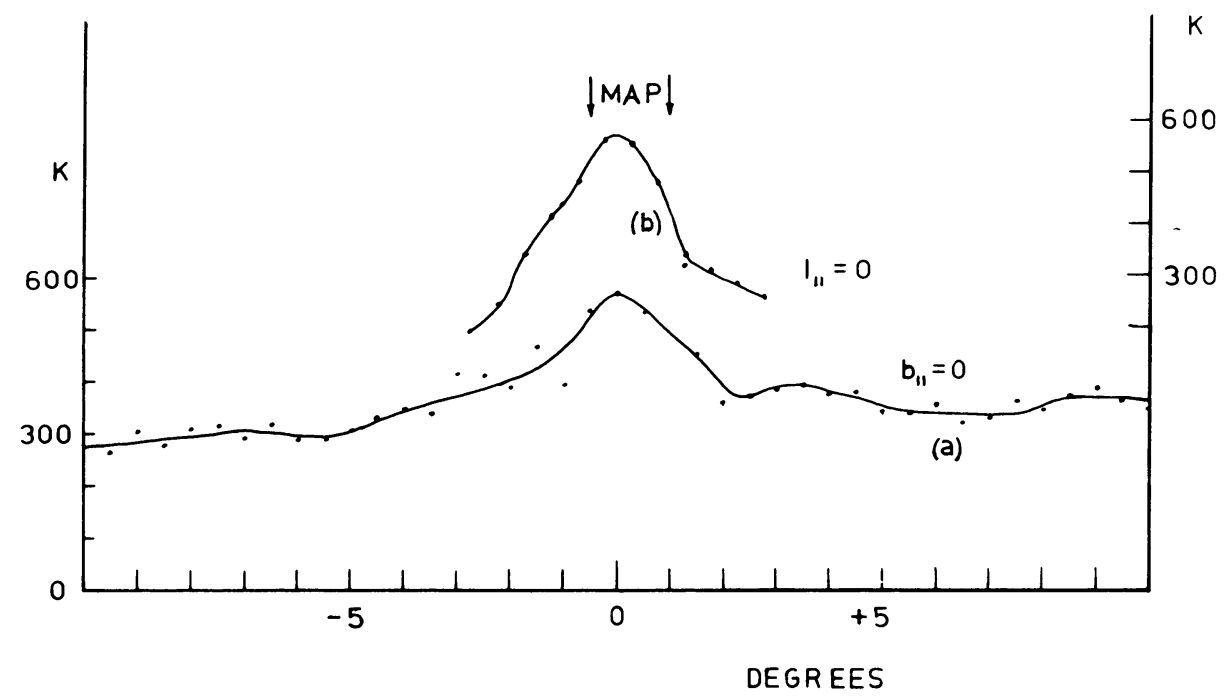

Fig. 3. The brightness temperature measured (a) along the plane at $b=0^{\circ}$ and (b) across the plane at $l=0^{\circ}$ with all the sources removed. The points are average values over $0.5^{\circ} \times 0.5^{\circ}$ cells.

the centre. Thus its peak temperature is obviously higher - possibly up to $40-50000 \mathrm{~K}$. Scaling this to $408 \mathrm{MHz}$ with a power law of $v^{-2.7}$ indicates a peak temperature of about $550-700 \mathrm{~K}$. The present $408 \mathrm{MHz}$ results are not too inconsistent with this, particularly if there is absorption at $408 \mathrm{MHz}$.

As Cooper and Price (1964) pointed out, there is a narrower source distribution approximately $1^{\circ} \times 0.5^{\circ}$ superimposed on that shown in Figure 3 . This distribution, which surrounds the Sgr A source, is clearly seen on the present map and has a maximum brightness temperature at $408 \mathrm{MHz}$ of $2100 \mathrm{~K}$. From the microwave results quoted by Downes and Maxwell (1966) the brightness temperature of this region should be $940 \mathrm{~K}$ at $408 \mathrm{MHz}$, assuming a simple thermal spectrum. Allowing for the $500 \mathrm{~K}$ nonthermal source just discussed, there is about $1600 \mathrm{~K}$ to be accounted for, which is more than the expected $940 \mathrm{~K}$ from the microwave results. This source may then be not purely thermal.

\section{Acknowledgements}

The author would like to acknowledge the help of Dr David Clark for producing the diagram of the line scans of Figure 1 and Dr A. Green for making available previously unpublished data and Professor B. Y. Mills for many helpful discussions.

\section{References}

Bracewell, R. N.: 1955, Australian J. Phys. 8, 200.

Broten, N. W., Cooper, B. F. C., Gardner, F. F., Minnett, H. C., Price, R. M., Tonking, F. G., and Yabsley, D. E.: 1965, Australian J. Phys. 18, 85.

Cooper, B. F. C. and Price, R. M.: 1964, in F. J. Kerr and A. W. Rodgers (eds.), 'The Galaxy and the Magellanic Clouds', IAU Symp. 20, 168. 
Downes, D. and Maxwell, A.: 1966, Astrophys. J. 146, 653.

Green, A.: 1974, Astron. Astrophys. Suppl. in press.

Hollinger, J. P.: 1965, Astrophys. J. 142, 609.

Hunstead, R. W.: 1972, Monthly Notices Roy. Astron. Soc. 157, 367.

Maxwell, A. and Taylor, J. H.: 1968, Astrophys. Letters 2, 191.

Mills, B. Y.: 1956, Observatory 76, 65.

Mills, B. Y., Aitchison, R. E., Little, A. G., and McAdam, W. B.: 1963, Proc. Inst. Radio Engineers (Australia) 24, 156.

Wyllie, D. V.: 1969a, Monthly Notices Roy. Astron. Soc. 142, 229.

Wyllie, D. V.: 1969b, Proc. Astron. Soc. Australia 1, 235.
A. G. Little
Chatterton Astronomy Department,
School of Physics,
University of Sydney,
Sydney, N.S.W., Australia

(Discussion follows the paper by Swarup et al., p. 508.) 